\title{
Cachexia-skeletal muscle depletion is a prognostic factor
}

It has been known for a long time that involuntary weight loss is prognostic of reduced survival in patients with cancer. What has not been clear is which tissue type might have been lost. Although obesity seems to confer a survival advantage in patients with cancer, previous research by Vickie Baracos and colleagues showed sarcopenia (severe muscle depletion) to be independently prognostic of poor survival in obese patients with cancer. Her team has now extended these findings to reveal that muscle depletion and low muscle attenuation are poor prognostic factors, regardless of overall body weight.

"We analysed CT images to quantify the amount of muscle and adipose tissue. CT imaging allows us to identify two characteristics of muscle: extreme depletion of skeletal muscle and reduced radiation attenuation of muscle." The present study that assessed over 1,400 patients, showed that obese patients who had weight loss, sarcopenia and low muscle attenuation had a significantly worse survival, which was in direct contrast to the survival of obese patients without these variables.

Baracos explains the relevance of these findings: "Ronenn Roubenoff is quoted as saying that we exist at the 'confluence of two epidemics'-epidemic obesity and epidemic muscle wasting, and I think that is a very apt description of what we have seen in our study." Baracos's team plans to move

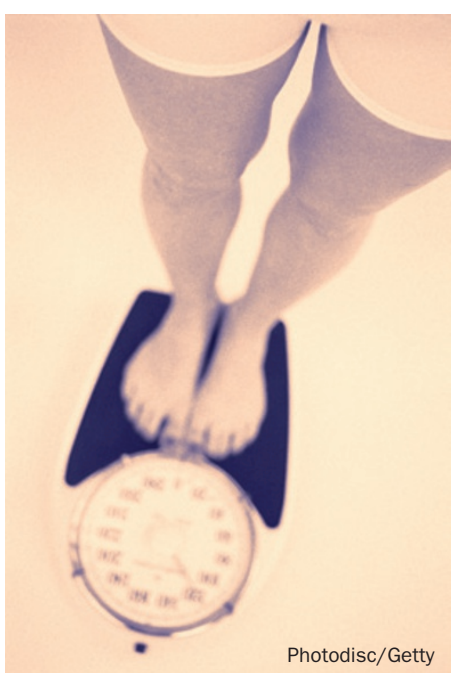

their latest research forward, with a two-pronged approach. They will first evaluate therapeutic interventions to prevent or reverse muscle wasting, which include muscle-specific anabolic agents and nutrients essential for the retention or gain of muscle mass. Baracos continues, "second, our analysis as well as a series of related works suggest that patients with severe depletion of skeletal muscle may be unusually prone to developing serious chemotherapy-related toxicity. We are evaluating approaches to chemotherapy dosing that will be consistent with the reduced tolerance of these individuals."

\section{Lisa Hutchinson}

Original article Martin, L. et al. Cancer cachexia in the age of obesity: skeletal muscle depletion is a powerful prognostic factor, independent of body mass index. J. Clin. Oncol. doi:10.1200/JC0.2012.45.2722 